\title{
Diagnóstico, estabilización y tratamiento del síndrome inflamatorio multisistémico pediátrico vinculado a SARS-CoV-2 (SIM-PedS)
}

\author{
Diagnosis, stabilization and treatment of SARS-CoV-2-linked \\ pediatric multisystemic inflammatory syndrome (SIM-PedS)
}

Alberto García-Salido, * Jordi Antón, ${ }^{\ddagger}$ José David Martínez-Pajares, ${ }^{\S}$

Gemma Giralt-García," Borja Gómez-Cortés," Alfredo Tagarro,** Grupo de trabajo de la Asociación

Española de Pediatría para el Síndrome Inflamatorio Multisistémico Pediátrico vinculado a SARS-CoV-2

\footnotetext{
* Sociedad Española de Cuidados Intensivos Pediátricos (SECIP). Cuidados Intensivos Pediátricos, Hospital Infantil Universitario Niño Jesús, Madrid, España.

‡ Sociedad Española de Reumatología Pediátrica (SERPE). Hospital Sant Joan de Déu, Institut de Recerca Sant Joan de Déu (IRSJD), Barcelona, España § Sociedad Española de Pediatría Hospitalaria (SEPHO). Unidad de Pediatría, Hospital de Antequera, Área Sanitaria Norte de Málaga, Málaga, España.

" Sociedad Española de Cardiología Pediátrica y Cardiopatías Congénitas (SECPCC). Hospital Universitario Hospital Vall d’Hebron, Barcelona, España.

"I Sociedad Española de Urgencias de Pediatría (SEUP). Servicio de Urgencias de Pediatría. Hospital Universitario Cruces, Barakaldo.

** Sociedad Española de Infectología Pediátrica (SEIP). Servicio de Pediatría, Hospital Universitario Infanta Sofía, Unidad Pediátrica de Investigación y Ensayos Clínicos (UPIC), Instituto de Investigación Sanitaria Hospital 12 de Octubre (IMAS12), Madrid, España.
}

\section{RESUMEN}

Se ha descrito un nuevo síndrome inflamatorio multisistémico pediátrico vinculado a SARS-CoV-2. Este cuadro presenta una expresividad clínica variable y se asocia a infección activa o reciente por SARS-CoV-2. En este documento se revisa la literatura existente por parte de un grupo multidisciplinar de especialistas pediátricos. Posteriormente, se realizan recomendaciones sobre estabilización, diagnóstico y tratamiento de este síndrome.

Palabras clave: Síndrome inflamatorio, niños, COVID-19, MIS-C, PIMS.

\section{INTRODUCCIÓN}

La infección por el nuevo coronavirus (SARS-CoV-2) en población pediátrica cursa, por lo general, de manera leve..$^{1,2}$ En España, los pacientes pediátricos menores de 15 años han supuesto un $0.4 \%$ de los ingresos hospitalarios, un $0.7 \%$ de los ingresados en cuidados intensivos y un 0.15 por 1,000 de los fallecidos. ${ }^{1}$ A principios de mayo del 2020 se describe en

Financiamiento: Ninguno.

Conflicto de intereses: Ninguno.

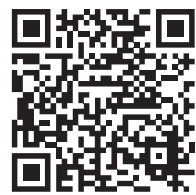

Rev Latin Infect Pediatr. 2021; 34 (1): 6-16

\section{ABSTRACT}

A new pediatric multisystemic inflammatory syndrome linked to SARS-CoV-2 has been described. This picture presents a variable clinical expressivity and is associated with active or recent SARSCoV-2 infection. This paper reviews the existing literature by a multidisciplinary group of pediatric specialists. Subsequently, recommendations on stabilization, diagnosis and treatment of this syndrome are made.

Keywords: Inflammatory syndrome, children, COVID-19, MIS-C, PIMS.

Europa un número limitado de niños con un síndrome inflamatorio sistémico de expresividad variable. ${ }^{3}$ Este síndrome muestra rasgos clínicos y analíticos similares a los observados en la enfermedad de Kawasaki (EK), el síndrome de choque tóxico (SST) o síndromes de activación macrofágica (SAM). ${ }^{4-7}$

Este nuevo síndrome parece asociarse con infección activa o reciente por SARS-CoV-2, en general en las últimas cuatro a seis semanas. La mayoría

Citar como: García-Salido A, Antón J, Martínez-Pajares JD, Giralt-García G Gómez-Cortés B, Tagarro A et al. Diagnóstico, estabilización y tratamiento del síndrome inflamatorio multisistémico pediátrico vinculado a SARS-CoV-2 (SIM-PedS). Rev Latin Infect Pediatr. 2021; 34 (1): 6-16. https://dx.doi. org/10.35366/99821

www.medigraphic.com/infectologiapediatrica 
Rev Latin Infect Pediatr. 2021; 34 (1): 6-16

presenta serología IgG positiva y aumento de biomarcadores de inflamación. Cerca de la mitad aún tienen PCR positiva, lo que sugiere una persistencia del antígeno viral, junto con disregulación inmunitaria. ${ }^{6,7}$

En este documento se utilizará la denominación «síndrome inflamatorio multisistémico pediátrico vinculado a SARS-CoV-2» o SIM-PedS para hacer referencia a este nuevo cuadro clínico.

\section{Criterios de sospecha clínica}

Se debe considerar esta enfermedad en áreas con alta incidencia actual o reciente de transmisión o enfermedad por SARS-CoV-2. La presencia de fiebre y afectación de dos o más órganos debe supo- ner una alerta para el clínico. Los criterios empleados según los diversos organismos se detallan en la Tabla 1 y los síntomas clínicos observados con mayor frecuencia en la Tabla 2.

Los cuadros clínicos que podrían ser compatibles, son los siguientes:

1. Cuadro clínico compatible con miocarditis, choque séptico o choque tóxico.

2. Cuadro clínico compatible con enfermedad de Kawasaki completa/incompleta.

3. Fiebre y dolor abdominal o exantema cutáneo o conjuntivitis, con alteración analítica compatible (especialmente reactantes de fase aguda

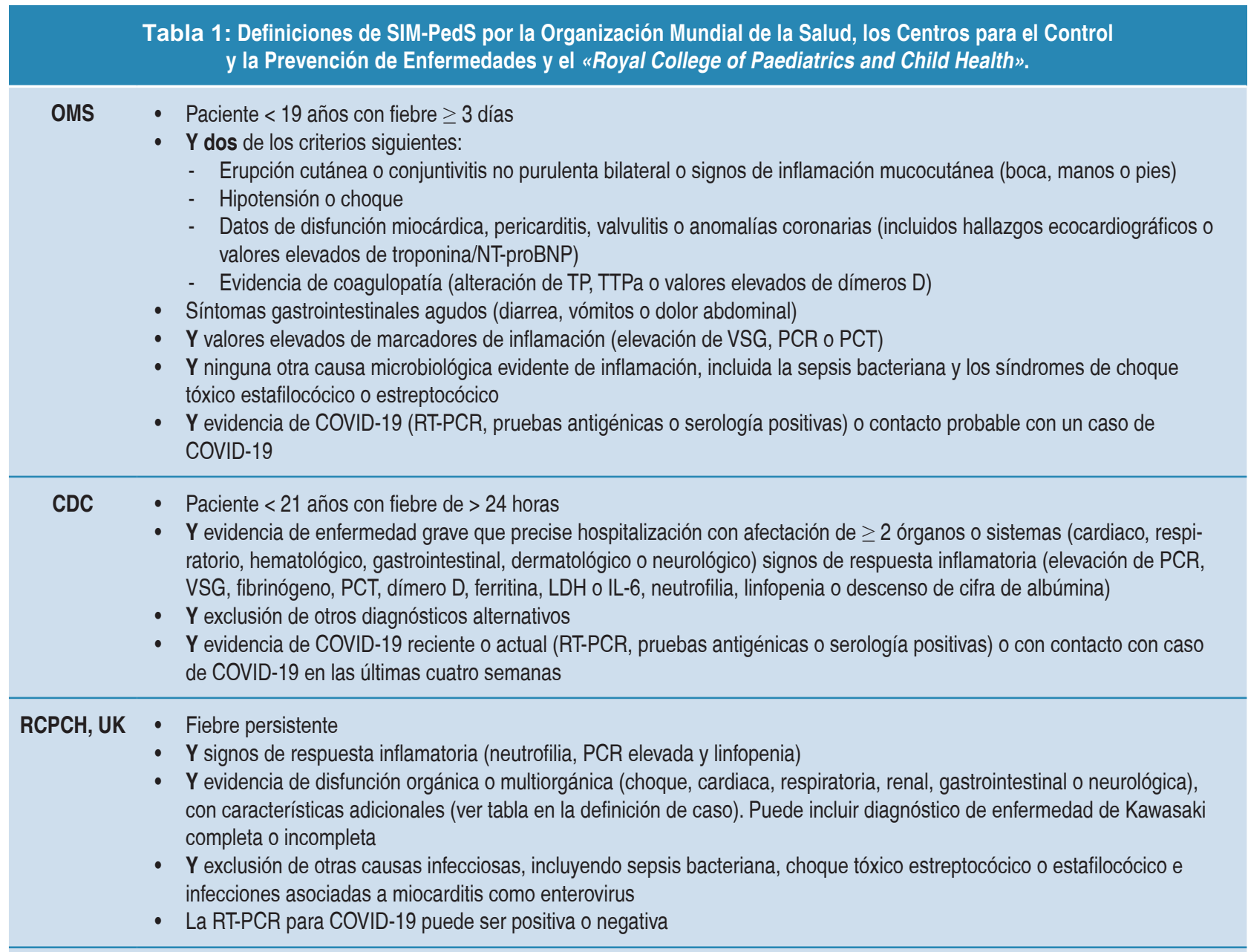

OMS = Organización Mundial de la Salud, $\mathrm{CDC}=$ Centros para el Control y la Prevención de Enfermedades, $\mathrm{RCPCH}=$ Royal College of Paediatrics and Child Health, TP = tiempo de protrombina, TTPa = tiempo de tromboplastina parcial activado, VSG = velocidad de sedimentación globular, $\mathrm{PCR}=$ proteína $\mathrm{C}$ reactiva, $\mathrm{PCT}=$ procalcitonina, IL-6 = interleucina 6 . 
Rev Latin Infect Pediatr. 2021; 34 (1): 6-16

Tabla 2: Datos clínicos y analíticos

observados con mayor frecuencia.

Datos clínicos

- Fiebre casi $100 \%$ de los casos; fiebre $>3$ días (una fiebre de corta evolución no lo descarta)

- Síntomas digestivos (> 50\%): dolor abdominal, vómitos, diarrea.

- Exantema (eritrodermia escarlatiniforme, eritema multiforme, livedo reticularis), conjuntivitis no exudativa, alteraciones mucosas, edemas periféricos (>2/3 de los pacientes)

- Choque, taquicardia, hipotensión, hipoperfusión (alrededor de la mitad de los pacientes)

- Cefalea, meningismo, confusión (10-20\%)

- Síntomas respiratorios: tos, disnea (10-20\%)

Datos analíticos

- Hemograma: Leucocitosis con linfopenia, neutrofilia y plaquetopenia

- Marcadores de inflamación: elevación de PCR, VSG, ferritina, fibrinógeno, LDH, IL-6. Procalcitonina normal o elevada (en ausencia de infección bacteriana)

- Coagulación: fibrinógeno, D-dímero elevado

- Bioquímica: hiponatremia, hipoalbuminemia, elevación de transaminasas (ALT, AST)

- Marcadores cardiacos: NT Pro-BNP muy elevado (>200 ng/L), elevación de enzimas miocárdicas (troponina-I, CPK-MB)

o RFA muy elevados y/o alteración de enzimas cardiacas).

Todo paciente con sospecha de SIM-PedS será trasladado a un centro hospitalario que permita su abordaje multidisciplinar. En general, se recomienda que los casos graves se trasladen a un centro con UCIP. El traslado se realizará en ambulancia medicalizada o UCI móvil en función de la situación del paciente.

\section{Diagnóstico diferencial}

Son de obligada consideración las siguientes enfermedades:

1. Sepsis de causa bacteriana.

2. Otras infecciones víricas (adenovirus, enterovirus, sarampión en población no inmunizada).

3. Abdomen agudo simulando peritonitis/apendicitis.

4. Síndrome de choque tóxico estreptocócico o estafilocócico.

5. Miocarditis por otros microorganismos.

6. Enfermedad de Kawasaki no relacionada con SARS-CoV-2.
7. Reacción de hipersensibilidad a fármacos (síndrome de Stevens-Johnson).

8. Otras enfermedades reumatológicas sistémicas (artritis idiopática juvenil sistémica y otras enfermedades autoinflamatorias o autoinmunes).

9. Linfohistiocitosis hemofagocítica primaria o secundaria (síndrome de activación macrofágica).

\section{ASISTENCIA Y ESTABILIZACIÓN INICIAL}

La asistencia y la estabilización inicial se basarán en la aproximación $A B C D E$, la vía aérea habitualmente se encontrará permeable salvo alteración del nivel de conciencia. Se administrará oxígeno suplementario acorde a las necesidades del paciente. Se monitorizará la saturación de oxígeno $\left(\mathrm{SatO}_{2}\right)$ y, si está disponible, el dióxido de carbónico espirado $\left(\mathrm{EtCO}_{2}\right)$. Se preparará material y medicación para secuencia rápida de intubación. En caso de requerir altas concentraciones de oxígeno, se consultará con una Unidad de Cuidados Intensivos Pediátricos (UCIP) o se valorará traslado a un centro con este servicio. Se monitorizarán constantes vitales.

\section{PRUEBAS COMPLEMENTARIAS}

No vinculadas a SARS-CoV-2. Se deben descartar otras causas infecciosas del cuadro clínico. En la Tabla 3 se muestran las pruebas complementarias recomendadas y las alteraciones más habituales en pacientes con sospecha de SIM-PedS..$^{10,11}$ Se recomienda la realización de un hemograma, bioquímica sanguínea, gasometría con lactato, coagulación (incluyendo dímero-D o DD), estudio de biomarcadores de infección en inflamación y estudio de marcadores de función miocárdica (ver más adelante). Además, se deben descartar otras causas infecciosas potencialmente causantes del cuadro clínico, por lo que se recomienda hemocultivo, PCR en aspirado/lavado nasal para antígenos o PCR de patógenos respiratorios y despistaje de otras infecciones según clínica.

De forma general, los siguientes parámetros analíticos se han relacionado con peor pronóstico: linfopenia, anemia y trombocitopenia, con valores más elevados de ferritina, LDH y dímero D (DD) se han observado en pacientes con choque. En el caso de los aneurismas coronarios, se han descrito leucocitosis y linfopenia más intensas con elevación de proteína $C$ reactiva (PCR). 
En comparación con otros cuadros clínicos de inflamación sistémica similares, como la EK o el síndrome de choque tóxico, los pacientes con SIM-PedS presentan habitualmente mayores elevaciones de los parámetros inflamatorios (mayores elevaciones de PCR, ferritina, citoquinas inflamatorias y NT-Pro-BNP, y con una mayor frecuencia de linfopenia y trombopenia, sin diferencias en los niveles de DD).

Vinculadas a SARS-CoV-2. Se recomienda como mínimo una toma de muestra respiratoria para realización de RT-PCR de SARS-CoV-2 (o un test antigénico). En caso de resultar negativa de inicio, y si existe una sospecha clínica elevada, se recomienda repetir en las 24-48 horas siguientes. En el caso de empeoramiento clínico que suponga la necesidad de ventilación mecánica invasiva, se recomienda obtener muestra de aspirado traqueal para RT-PCR.

Además, se recomienda realizar al menos una serología tanto en casos con RT-PCR positiva como negativa. El rendimiento será superior una vez pasados un mínimo de diez días desde el primer signo o síntoma. En el caso de RT-PCR negativa con serología negativa y alta sospecha clínica se recomienda repetir serología de tres a cuatro semanas después del ingreso hospitalario (entre un $26-55 \%$ de los pacientes con SIM-PedS tienen RT-PCR positiva y hasta un $90 \%$ serología lgG positiva).

\section{Pruebas de imagen}

La radiografía o ecografía torácica pueden estar indicadas en caso de sintomatología respiratoria, exploración patológica o para localizar dispositivos (tubo endotraqueal o catéter central). La radiografía de tórax puede mostrar alteraciones en cerca de la mitad de los casos. Se han descrito infiltrados, condensaciones parcheadas, en vidrio esmerilado o patrón intersticial, atelectasias o derrame pleural. En la ecografía torácica se pueden observar patrones de neumonía o aumento del agua pulmonar.

La tomografía computarizada (TC) torácica no se recomienda de rutina, excepto en neumonía complicada, mala evolución o en paciente inmunocomprometido.

Se realizará ecografía o TC abdominal si existe clínica abdominal sugestiva de abdomen agudo para detectar complicaciones y clarificar la necesidad de cirugía. Está descrita la presencia de ileocolitis.

Tabla 3: Pruebas complementarias recomendadas y alteraciones más habituales.

\begin{tabular}{|c|c|}
\hline Prueba & Alteración \\
\hline Hemograma & $\begin{array}{l}\text { - Leucocitosis (habitualmente }<20.000 / \mathrm{mm}^{3} \text { ) con linfopenia } \\
\text { - Anemia variable } \\
\text { - Trombopenia leve }\left(\text { normalmente }>50.000 / \mathrm{mm}^{3}\right)\end{array}$ \\
\hline lonograma & - Hiponatremia \\
\hline Bioquímica hepática y albúmina & $\begin{array}{l}\text { - Aumento de transaminasas } \\
\text { - Hipoalbuminemia }\end{array}$ \\
\hline Bioquímica cardiaca* & - Aumento de BNP (>35 pg/ml) o NT-proBNP (>125 pg/mL) y troponina ultrasensible (>14ng/L). \\
\hline Gasometría & - Acidosis metabólica/respiratoria, según estado clínico \\
\hline Marcadores inflamatorios* & - Elevación de PCR (> $20 \mathrm{mg} / \mathrm{dL})$, PCT (>0.5 $\mu \mathrm{g} / \mathrm{mL}), \mathrm{IL}-6$ (> $8.5 \mathrm{pg} / \mathrm{mL})$ y ferritina (> $120 \mathrm{mg} / \mathrm{dL}$ ) \\
\hline Estudio de coagulación & $\begin{array}{l}\text { - Aumento de fibrinógeno (> } 400 \mathrm{mg} / \mathrm{dL}) \\
\text { - } \text { Aumento significativo del dímero-D }(>500 \mathrm{ng} / \mathrm{mL}) \\
\text { - TP/TPPA alterados }\end{array}$ \\
\hline Hemocultivo & - Generalmente negativos \\
\hline Otros & $\begin{array}{l}\text { - Considerar la presencia de coinfecciones } \\
\text { - Urocultivo } \\
\text { - Cultivo de heces/PCR patógenos en heces }\end{array}$ \\
\hline
\end{tabular}

* Valores orientativos, considerar los de referencia para cada centro hospitalario. PCR = proteína $\mathrm{C}$ reactiva, TP/TPPA = tiempo de protrombina $/$ tiempo de tromboplastina parcial activado, PCT = procalcitonina. 


\author{
Pruebas complementarias vinculadas \\ a función cardiaca
}

Electrocardiograma: inespecífico, puede mostrar alteraciones sugestivas de afectación miocárdica como voltajes bajos, anomalías del segmento ST y onda T y/o intervalo QTc prolongado. Se han descrito diversos grados de bloqueo auriculoventricular, arritmias supraventriculares y ventriculares.

Marcadores de daño miocárdico: un alto porcentaje de estos pacientes presenta elevación de troponina I o T (55-68\%) y BNP/NT-proBNP (83-100\%), con cifras significativamente mayores en aquéllos que desarrollan choque.

Ecocardiografía: se realizará ecocardiografía en las primeras 24 horas de ingreso a todos los pacientes con SIM-PedS. En formas leves no son habituales las alteraciones ecocardiográficas. En cuadros graves se ha descrito, sobre todo, disfunción sistólica del ventrículo izquierdo. También se ha descrito disfunción del ventrículo derecho, insuficiencia mitral, derrame pericárdico y dilatación o aneurismas de las arterias coronarias (AC). La evaluación de las AC debería incluir la determinación del Z-score y clasificación según el Consenso Nacional de la Enfermedad de Kawasaki.

Resonancia magnética cardiaca: no indicada en periodo agudo.

\section{ACTUACIÓN EN PLANTA DE HOSPITALIZACIÓN}

Identificación y acogida: recepción en planta con identificación, recogida de constantes (incluido el peso), comprobación de dispositivos (vías, sondajes, etcétera) y medicación.

Medidas de aislamiento: un elevado porcentaje de pacientes tendrán una prueba RT-PCR negativa y serología con IgG positiva. Esto indicaría una baja o nula contagiosidad. En casos de posible infección activa aún con RT-PCR negativa, se recomendarán las siguientes medidas de aislamiento habituales hasta descartar contagiosidad.

Monitorización: se recomienda pulsioximetría continua (frecuencia cardiaca y $\mathrm{SatO}_{2}$ ) y determinación de diuresis y TA, al menos por turno.

\section{Tratamiento de soporte}

Antitérmicos de primera elección paracetamol oral o intravenoso. Se considerará en caso de persistencia de fiebre o respuesta parcial la aplicación de metamizol intravenoso $u$ oral.

Para fluidoterapia se preconizará el uso de sueros isotónicos o balanceados. Se deben vigilar signos de deshidratación, sobrecarga hídrica o intolerancia oral.

Antibioterapia con base en sospecha clínica y protocolos del centro; oxigenoterapia si precisa.

Tratamiento antiagregante y anticoagulante en pacientes seleccionados (ver apartado de tratamiento antitrombótico).

En cuanto a la valoración cardiológica, se recomienda realizar ecocardiografía, electrocardiograma (ECG), biomarcadores de lesión cardiaca (troponinas) y BNP o NT-proBNP en las primeras 12 horas de ingreso. Se repetirán si no se observa mejoría clínica o no hay respuesta al tratamiento realizado.

Seguimiento del paciente. Estudio normal: ECG y marcadores a las 72 horas, y valorar ecografía en una a dos semanas. Además se repetirá ecocardiografía a las seis semanas del diagnóstico.

Elevación de marcadores de lesión cardiaca: repetir estudio a las 24 horas.

Dilatación coronaria: repetir ecografía, ECG y marcadores cada dos a tres días, hasta que el tamaño de las coronarias se normalice o estabilice, con seguimiento posterior según el Consenso Nacional de EK. Si aparecen aneurismas gigantes, valorar realizar angioTC de coronarias.

ECG diario si se utiliza medicación que pueda prolongar el intervalo QTC.

\section{Criterios de traslado e ingreso en UCIP}

1. Inestabilidad hemodinámica a pesar de expansión de volemia, con hipotensión arterial o signos de hipoperfusión clínicos o gasométricos (acidosis metabólica, hiperlactacidemia).

2. Requerimiento de soporte vasoactivo.

3. Afectación miocárdica comprobada por ecocardiografía.

4. Alteración del estado de conciencia sin mejoría a pesar de tratamiento optimizado.

5. Requerimiento de soporte respiratorio superior a oxigenoterapia en gafas nasales. En caso de no mejoría en un periodo nunca superior a las ocho horas, valorar traslado. El uso de asistencia con alto flujo en cánulas nasales en planta de hospitalización, dado el rápido empeoramiento que pueden presentar estos pacientes, no es recomendable y se debe interpretar como un signo de empeoramiento y traslado. 
6. Signos de disfunción de dos o más órganos que no responden o que no muestran mejoría a pesar de tratamiento sintomático.

\section{ACTUACIÓN EN CUIDADOS INTENSIVOS PEDIÁTRICOS}

Consideraciones generales. En caso de ingreso en UCIP, el paciente permanecerá en una habitación aislada, preferentemente con presión negativa. Si se requieren maniobras que produzcan aerosoles, se utilizará mascarilla FFP3, gafas de protección integral, monointegral o capucha, bata desechable impermeable, si no se producen aerosoles, con delantal impermeable y guantes. En caso de intubación, se utilizará también doble guante.

El paciente estará acompañado de un familiar o cuidador que seguirá las medidas de aislamiento e higiene que se le indiquen. Usará, como mínimo, mascarilla quirúrgica, bata y guantes. En función de los resultados de RT-PCR y serología, se valorará modificar las medidas de protección, manteniendo siempre aislamiento por contacto y gotas.

Se añadirá la monitorización necesaria en función de su situación y gravedad. En los casos graves, se valorará realizar una canalización arterial y venosa central precoces.

Soporte respiratorio. Aunque estos pacientes suelen precisar de un soporte respiratorio $(70 \%)$, habitualmente es debido a la repercusión cardiaca y/o hemodinámica existente.

Se administrará oxigenoterapia para mantener $\mathrm{SatO}_{2}$ entre $94-98 \%$ mediante gafas nasales cubiertas con mascarilla quirúrgica.

Si por la situación clínica o gasométrica la oxigenoterapia no fuera suficiente, se valorará el uso de oxigenoterapia de alto flujo (OAF) o de ventilación no invasiva (VNI), aunque son procedimientos que pueden generar aerosoles, por lo que siempre debe hacerse con las medidas de aislamiento y protección adecuadas. Si hay hipoxemia sin hipercapnia, considerar iniciar OAF.

Si la OAF no es efectiva, como alternativa a ésta se puede iniciar VNI con CPAP, con mascarilla buconasal, facial total o Helmet (este último es el modo más estanco). Si existe hipercapnia asociada, valorar el uso de BIPAP.

Si no hay una mejoría clara de los parámetros clíni$\cos (\mathrm{FC}, \mathrm{FR}$, dificultad respiratoria) y de oxigenación $\left(\mathrm{PaO}_{2} / \mathrm{FiO}_{2}, \mathrm{SatO}_{2} / \mathrm{FiO}_{2}\right)$ en las primeras horas del soporte con OAF o VNI, se recomienda intubación precoz y ventilación mecánica invasiva. Además, se valorará intubación precoz si existe deterioro del estado de conciencia o choque refractario a fluidos o resistente a catecolaminas.

En caso de intubación, preoxigenar con $\mathrm{O}_{2}$ al $100 \%$ con mascarilla facial durante cinco minutos, evitando en lo posible la ventilación balón-mascarilla y realizando el procedimiento con las medidas de protección recomendadas.

Se recomiendan los siguientes parámetros iniciales: VT 4-8 mL/kg, búsqueda de PEEP óptima para lograr un reclutamiento adecuado, presiones mesetas $<30 \mathrm{~cm} \mathrm{H}_{2} \mathrm{O}$ y drive pressure $<15 \mathrm{~cm} \mathrm{H}_{2} \mathrm{O}$. Si evoluciona a un síndrome de distrés respiratorio agudo (SDRA) moderado-severo, se recomienda intubación precoz, siguiendo las recomendaciones de ventilación protectora arriba indicadas, con hipercapnia permisiva, uso de prono y de bloqueo neuromuscular si es preciso. Se reserva el óxido nítrico para situaciones de hipoxemia refractaria, especialmente si está asociada a hipertensión pulmonar.

Soporte hemodinámico. La inestabilidad hemodinámica se encontrará habitualmente relacionada con choque vasopléjico y/o disfunción cardiaca. En caso de marcadores elevados inflamatorios, troponina y NT pro-BNT elevados o alteraciones electrocardiográficas, se recomienda siempre una evaluación ecocardiográfica precoz.

Si presenta hipotensión arterial o signos de hipoperfusión, (con o sin taquicardia), se realizará expansión de volumen de $10-20 \mathrm{~mL} / \mathrm{kg}$ vigilando estrechamente signos de sobrecarga de volumen.

Se considerará choque refractario a líquidos si la hipotensión persiste tras dos expansiones de volumen (hasta $40 \mathrm{~mL} / \mathrm{kg}$ ), con especial precaución con la sobrecarga hídrica si tiene disfunción cardiaca.

Se iniciará apoyo inotropo/vasopresor según el tipo de choque, inicialmente con adrenalina o noradrenalina (ésta preferentemente si es vasopléjico).

En la disfunción cardiaca, además de adrenalina, valorar asociar milrinona o si es moderada/grave, levosimendán.

Si hay una hipotensión refractaria, realizar tratamiento vasopresor con noradrenalina o adrenalina a dosis más altas (>0.2 $\mu \mathrm{g} / \mathrm{kg} / \mathrm{min})$. Si persiste hipotensión, añadir corticoides (si no se han iniciado ya) y considerar administrar vasopresina.

Si aparecen arritmias, tratarlas de forma inmediata según el tipo y el deterioro clínico que producen (valorar amiodarona o cardioversión en casos graves). 


\section{TRATAMIENTO FARMACOLÓGICO}

Tratamiento antibiótico: en todos los casos se realizará si existe sospecha de infección bacteriana, sin retrasarlo a pesar de considerar que el paciente presente un SIM-PedS. Se aplicarán las recomendaciones y protocolos de cada centro hospitalario adecuados a la edad y foco infeccioso considerados como más probables.

Tratamiento inmunomodulador: se debe usar de forma escalonada, constituyendo la primera línea el uso de inmunoglobulina intravenosa (IGIV) y/o corticoides. En casos graves o sin respuesta, se recomienda combinarlos.

Inmunoglobulina intravenosa: se propone el uso de IGIV en dosis de $2 \mathrm{~g} / \mathrm{kg}$, sobre todo en aquellos casos que cumplen criterios de EK o de síndrome de choque tóxico. En caso de inestabilidad hemodinámica se podrá administrar $1 \mathrm{~g} / \mathrm{kg} /$ día durante dos días. Se consideraría la administración de una segunda dosis a las 36 horas de la finalización de la primera ante la persistencia de fiebre.

Corticoides sistémicos: se propone la administración de corticoides intravenosos, tanto como primer escalón, como para aquellos pacientes que no respondan a una primera dosis de IGIV. Se debe considerar su uso precoz ante factores de riesgo para la presencia de aneurismas coronarios o criterios analíticos compatibles con SAM.

Se proponen tres opciones de administración:

1. Formas leves-moderadas: metilprednisolona intravenosa 1-2 mg/kg/día durante tres a cinco días y suspender. En aquellos casos que requieren de un tratamiento de seis o más días o bien persiste clínica o biomarcadores de inflamación elevados, se realizará paso posterior a prednisona oral y reducción progresiva en dos a tres semanas.

2. Formas graves (choque, especialmente si requieren altas dosis de inotrópicos/vasopresores): metilprednisolona intravenosa 1-2 mg/ $\mathrm{kg} / \mathrm{día}$ durante tres a cinco días o metilprednisolona intravenosa $30 \mathrm{mg} / \mathrm{kg} /$ día durante uno a tres días (máximo un gramo). Se continuará en caso de buena respuesta con prednisona oral a 1-2 mg/kg/día, con disminución progresiva hasta normalización de los parámetros inflamatorios.

3. Formas Kawasaki-like: IGIV $2 \mathrm{~g} / \mathrm{kg}$. Asociar corticoides conjuntamente con las IGIV en los pacientes de alto riesgo de resistencia a IGIV (varón, menor de 12 meses, PCR mayor de 100 $\mathrm{mg} / \mathrm{L}$, plaquetas menores de $300.000 / \mathrm{mm}^{3}, \mathrm{ALT}$ mayor de $100 \mathrm{UI} / \mathrm{L}$, neutrofilia superior al $80 \%$, sodio menor de $133 \mathrm{mmol} / \mathrm{L}$ ).

En caso de falta de respuesta a IGIV y corticoides, se ha propuesto el tratamiento con fármacos inmunosupresores selectivos (biológicos). Es importante que en los equipos que tratan estos pacientes se incluyan especialistas pediátricos con experiencia en la utilización de estos fármacos.

Bloqueo de IL-1 (anakinra): se ha utilizado con éxito en la neumonía grave con hiperinflamación por SARS-CoV-2, SAM y en EK refractaria a IGIVI corticoides. Con base en esto podría ser de utilidad en SIM-PedS. Su vida media corta, su rapidez de acción y la escasa asociación a sobreinfecciones bacterianas, lo postulan como un fármaco seguro. Aunque se presenta en jeringa para administración subcutánea, se ha empleado vía intravenosa en casos graves. Se mantendría durante cinco a 14 días, según respuesta clínica.

Bloqueo IL-6: tocilizumab, aunque se ha empleado puntualmente en el SIM-PedS, el uso de tocilizumab en pacientes con EK podría acelerar la formación de aneurismas coronarios, por lo que no se considera de elección.

\section{Tratamiento antiviral o dirigido a disminuir la replicación viral}

En el momento de redactar este documento no se ha descrito evidencia en relación con la eficacia y seguridad de ningún fármaco dirigido contra el virus en la infancia. A pesar de que el papel del SARS-CoV-2 en el SIM-PedS no está claro, en caso de infección activa, alta sospecha o casos graves cabe plantearse el tratamiento con remdesivir. Su uso en pediatría está restringido y de momento sólo se consigue a través de ensayo clínico o como uso compasivo previa autorización.

Dosis recomendada: 2.5-40 kg. Dosis carga: 5 $\mathrm{mg} / \mathrm{kg} / 24$ horas (una dosis). Dosis mantenimiento: $2.5 \mathrm{mg} / \mathrm{kg} / 24$ horas.

Dosis recomendada: $\geq 40 \mathrm{~kg}$. Dosis carga: 200 $\mathrm{mg} / 24$ horas (una dosis). Dosis mantenimiento: 100 $\mathrm{mg} / 24$ horas.

Monitorizar efectos secundarios: hipertransaminasemia y alteración de la función renal. 
Rev Latin Infect Pediatr. 2021; 34 (1): 6-16

\section{Tratamiento antitrombótico}

Hasta la fecha, hay pocos casos pediátricos con COVID-19 que hayan desarrollado complicaciones tromboembólicas, por lo que parece que el riesgo en niños es menor que el observado en adultos.

Se podría considerar individualmente la profilaxis con heparina de bajo peso molecular (enoxaparina) en caso de pacientes con enfermedad de base protrombótica o pacientes graves con imposibilidad de deambulación. En caso de enfermedad tromboembólica venosa habría que cambiar a dosis de tratamiento, con ajuste de la dosis según los niveles de anti-Xa.

En los siguientes casos se considerará el uso de heparina de bajo peso molecular:

1. Dímero $\mathrm{D}$ igual o superior a seis veces su valor normal.

2. Presencia de trombosis diagnosticada en el paciente.

3. Paciente inmovilizado.

4. Presencia de aneurismas gigantes.

5. Disfunción severa del ventrículo izquierdo (FE $<30 \%$ ).

6. Antecedentes personales o familiares de enfermedad tromboembólica.

7. Antecedentes personales de patología isquémica arterial (periférica, cardiaca o neurológica).

Pautas recomendadas:

Enoxaparina subcutánea $1 \mathrm{mg} / \mathrm{kg} / \mathrm{día}$ (si insuficiencia renal con filtrado glomerular menor de 30 $\mathrm{mL} / \mathrm{min} / \mathrm{m}^{2}$, se utilizará una dosificación de $0.25 \mathrm{mg} /$ $\mathrm{kg} / 12 \mathrm{~h}$ ). Control de anti-Xa a las 48-72 horas (nivel recomendado 0.3-0.49). Mantener hasta resolución del cuadro y hasta dímero $D$ normalizado según valores de referencia en el centro de ingreso.

Pacientes con tromboembolismo o sospecha de trombosis venosa profunda: enoxaparina $1 \mathrm{mg} / \mathrm{kg} /$ dosis cada 12 horas SC. En dosis de anticoagulación (0.5-1 de anti $\mathrm{Xa}$ ). Control de anti-Xa a las 48 horas (ajuste de dosis según referencia). Se mantendrá durante todo el ingreso realizándose consulta al servicio de hematología de forma previa al alta.

\section{Tratamiento ácido con acetilsalicílico}

Indicación antiinflamatoria: se utilizará en aquellos SIM-PedS que cumplan criterios de EK clásico o incompleto. Será indicado junto con la IGIV. Se debe iniciar tratamiento con ácido acetilsalicílico (AAS) a 30-50 mg/kg/día cada seis horas, vía oral, hasta que el paciente esté 48 horas afebril. En ese momento se disminuirá a dosis antiagregante, 3-5 mg/kg/día en una dosis oral. Se mantendrá esta dosis hasta seis a ocho semanas del inicio de la enfermedad y tras comprobar la normalización del recuento plaquetario, reactantes de fase aguda y ecocardiograma.

Indicación antiagregante: los SIM-PedS con afectación clínica grave, presencia de aneurismas, datos clínicos o analíticos de inflamación y/o trombocitosis superior a $700.000 / \mathrm{mm}^{3}$ se valorará AAS a dosis antiagregante durante seis semanas (confirmando ecocardiografía normal tras este periodo). Esta recomendación se realiza porque se han descrito alteraciones coronarias también en pacientes sin características de EK o EK.

\section{TRATAMIENTO DE RESCATE, ECMO}

Podrán ser candidatos a ECMO los casos con SIMPedS que presenten un cuadro clínico refractario a las medidas convencionales máximas, con situación que se prevé reversible y que no presentan contraindicaciones absolutas para ECMO.

Resultará fundamental derivar estos pacientes antes de que su condición haga imposible el transporte convencional.

\section{ALTA Y SEGUIMIENTO}

Se recomienda un seguimiento ambulatorio multidisciplinar y coordinado de síndrome clínico y sus consecuencias por los especialistas pediátricos hospitalarios más implicados, con la participación de los pediatras de atención primaria.

\section{Criterios de alta hospitalaria}

No existen criterios estandarizados globales para dar de alta a pacientes pediátricos con SIM-PedS, por lo que se debe considerar lo siguiente:

1. Afebril, al menos en las últimas $24-48$ horas.

2. Sin necesidad de oxígeno durante 12-24 horas (aire ambiente, $\mathrm{SpO}_{2}$ mayor o igual a $90-92 \%$ y sin signos de dificultad respiratoria).

3. Mejoría/normalización de las pruebas de imagen (radiografía de tórax, normalización de la función cardiaca). 
4. Mejoría analítica. Monitorizar y comprobar normalización o mejoría de linfopenia, reactantes de fase aguda, DD, IL-6 y biomarcadores cardiacos. Se puede considerar no repetir si es normal al ingreso y/o existe una clara mejoría clínica durante el mismo.

5. Ingesta oral adecuada.

6. Capacidad o posibilidad de completar el tratamiento en casa, si es necesario.

\section{RESUMEN DE RECOMENDACIONES}

Todo paciente con sospecha de SIM-PedS deberá ser ingresado en un centro hospitalario que permita un abordaje pediátrico multidisciplinar.

1. En la asistencia inicial se solicitará hemograma, coagulación, pruebas de función renal y hepática, enzimas cardiacas (troponina, BNP/ NT-ProBNP), gasometría, biomarcadores inflamatorios y estudio microbiológico no vinculados a coronavirus (cultivos y otros según clínica). Se añadirán pruebas de imagen en función de la afectación clínica o sospecha diagnóstica.

2. Se recomienda obtener una muestra respiratoria para realización de RT-PCR para SARS-CoV-2. Se recomienda realizar al menos un estudio serológico mediante técnica automatizada.

3. Siempre se realizará ecocardiografía en las primeras 24 horas de ingreso para valorar función cardiaca y afectación coronaria. Esta prueba se utilizará además para la determinación del tipo de choque y su tratamiento. En caso de disfunción miocárdica se recomienda el traslado a centro con Cuidados Intensivos Pediátricos.

4. Se mantendrán medidas de aislamiento para el paciente y sus cuidadores. Se establecerán medidas de protección para los profesionales hasta descartar infección activa por SARSCoV-2.

5. Se monitorizará estrechamente la situación hemodinámica y respiratoria. Ante empeoramiento significativo o no mejoría en un periodo nunca superior a ocho horas se considerará el traslado a un centro con Unidad de Cuidados Intensivos Pediátricos. Si es necesario el uso de oxigenoterapia de alto flujo en planta de pediatría se considerará siempre la posibilidad de traslado a UCIP o a hospital con UCIP. Previo al mismo se realizará su estabilización, basada en la aproximación ABCDE.
6. En cualquier momento de la asistencia se procederá al traslado ante un choque que no responde a expansión de volemia.

7. En los casos graves se iniciará tratamiento inotrópico y vasopresor de forma precoz. Se recomienda después monitorización invasiva y medición del gasto cardiaco.

8. El soporte respiratorio deberá ser proporcional a la situación clínica y hemodinámica. En el paciente grave la oxigenoterapia de alto flujo o la ventilación no invasiva pueden ser medidas de soporte adecuadas, cumpliendo con las medidas de protección frente a la producción de aerosoles.

9. Se realizará intubación precoz si hay un choque refractario a fluidos y/o catecolaminas o no existe una mejoría respiratoria con un soporte no invasivo en las primeras horas. En caso de requerir ventilación mecánica invasiva, se minimizará su repercusión hemodinámica y se utilizarán las estrategias habituales de ventilación protectora. En situación de inestabilidad refractaria al tratamiento, se valorará el traslado precoz a un centro con programa de ECMO.

10. El tratamiento inmunomodulador se pautará de forma escalonada. Se recomienda de primera elección el uso de IGIV y/o corticoides intravenosos. En pacientes con falta de respuesta IGIV o factores de riesgo de afectación de coronarias por signos y síntomas compatibles con EK, choque con o sin requerimiento de inotrópicos o síndrome de activación macrofágica se considerará siempre la administración de corticoides.

11. En el caso de infección activa por SARS-CoV-2, y considerando la gravedad del cuadro clínico, se considerará el uso de remdesivir.

12. Se considerará la profilaxis con heparina de bajo peso molecular en pacientes con factores de riesgo protrombóticos o pacientes graves con imposibilidad de deambulación. En caso de enfermedad tromboembólica venosa, se usará heparina de bajo peso molecular a dosis terapéuticas.

13. Se recomienda el uso de AAS a dosis antiagregante en aquellos cuadros de SIM-PedS con criterios de EK clásica/completa o incompleta. Se valorará su uso en los cuadros graves de SIM-PedS sin criterios de EK.

14. Se recomienda en todos los pacientes con SIM-PedS, incluidos aquéllos con coronarias 
Rev Latin Infect Pediatr. 2021; 34 (1): 6-16

no afectadas, seguimiento cardiológico con ecocardiografía a las dos y seis semanas del episodio agudo.

15. Se realizará seguimiento en función de la clínica del paciente por parte de los especialistas pediátricos implicados en la atención recibida o la infección por SARS-CoV-2.

\section{AGRADECIMIENTOS}

\section{Miembros del Grupo de trabajo de la \\ Asociación Española de Pediatría para \\ el Síndrome Inflamatorio Multisistémico \\ Pediátrico vinculado a SARS-CoV-2.}

Sylvia Belda-Hofheinz (Sociedad Española de Cuidados Intensivos Pediátricos (SECIP), Hospital Universitario 12 de Octubre de Madrid), Inmaculada Calvo-Penadés (Sociedad Española de Reumatología Pediátrica (SERPE), Hospital Universitario y Politécnico La Fe), Juan Carlos de Carlos-Vicente (Sociedad Española de Cuidados Intensivos Pediátricos (SECIP), Hospital Universitario Son Espases), Carlos Daniel Grasa-Lozano (Sociedad Española de Infectología Pediátrica (SEIP), Hospital Universitario La Paz), Susanna Hernández-Bou (Sociedad Española de Urgencias de Pediatría (SEUP), Hospital Sant Joan de Déu), Rosa M Pino-Ramírez (Sociedad Española de Pediatría Hospitalaria (SEPHO), Hospital Sant Joan de Déu), Esmeralda NúñezCuadros (Sociedad Española de Reumatología Pediátrica (SERPE), Hospital Regional Universitario de Málaga), Javier Pérez-Lescure-Picarzo (Sociedad Española de Cardiología Pediátrica y Cardiopatías Congénitas (SECPCC), Hospital Universitario Fundación Alcorcón), Jesús Saavedra-Lozano (Sociedad Española de Infectología Pediátrica (SEIP), Hospital General Universitario Gregorio Marañón), Diana Salas-Mera (Sociedad Española de Cardiología Pediátrica y Cardiopatías Congénitas (SECPCC), Hospital Universitario La Paz), Enrique VillalobosPinto (Sociedad Española de Pediatría Hospitalaria (SEPHO), Hospital Infantil Universitario Niño Jesús), María Bernardino (Universidad Europea de Madrid).

\section{REFERENCIAS}

1. García SA, Antón J, Martínez PJ et al. Spanish consensus document on diagnosis, stabilisation and treatment of pediatric multisystem inflammatory syndrome related to SARS-CoV-2 (SIM-PedS)]. An Pediatr (Barc).
2021; 94 (2): 116.e1-116.e11. Spanish. doi: 10.1016/j. anpedi.2020.09.005.

2. Multisystem inflammatory syndrome in children and adolescents with COVID-19. 2020. [Accessed June 16, 2020].

3. Rapid risk assessment: Paediatric inflammatory multisystem syndrome and SARS-CoV-2 infection in children. 2020. [Accessed 19 June 2020] Available in: https://www.ecdc. europa.eu/en/publications-data/paediatric-inflammatorymultisystem-syndrome-and-sars-cov-2-rapid-riskassessment.

4. Abdel-Mannan O, Eyre M, Lobel U et al. Neurologic and radiographic findings associated with COVID-19 infection in children. JAMA Neurol. 2020; 77 (11): 1440-1445.

5. McCrindle BW, Rowley AH, Newburger JW et al. Diagnosis, treatment, and long-term management of Kawasaki disease: a scientific statement for health professionals from the American Heart Association. Circulation. 2017; 135: e927-e99.

6. Whittaker E, Bamford A, Kenny J et al. Clinical characteristics of 58 children with a pediatric inflammatory multisystem syndrome temporally associated with SARS-CoV-2. JAMA. 2020; 324 (3): 259-269.

7. Weiss SL, Peters MJ, Alhazzani W et al. Surviving sepsis campaign international guidelines for the management of septic shock and sepsis-associated organ dysfunction in children. Pediatr Crit Care Med. 2020; 21: e52-e106.

8. Ramcharan T, Nolan O, Lai CY et al. Paediatric inflammatory multi-system syndrome-temporally associated with SARSCoV-2 (PIMS-TS): cardiac features, management and shortterm outcomes at a UK tertiary paediatric hospital. Pediatr Cardiol. 2020; 12: 1-11.

9. Belhadjer Z, Meot M, Bajolle F et al. Acute heart failure in multisystem inflammatory syndrome in children (MIS-C) in the context of global SARS-CoV-2 pandemic. Circulation. 2020; 142 (5): 429-436.

10. Verdoni L, Mazza A, Gervasoni A et al. An outbreak of severe Kawasaki-like disease at the Italian epicentre of the SARS-CoV-2 epidemic: an observational cohort study. Lancet (London, England). 2020; 395: 1771-1778.

11. Kaushik S, Aydin SI, Derespina KR et al. Multisystem inflammatory syndrome in children (MIS-C) associated with SARS-CoV-2 infection: a multi-institutional Study from New York City. J Pediatr 2020; 224: 24-29.

12. Riphagen S, Gomez X, Gonzalez-Martinez C, Wilkinson N, Theocharis P. Hyperinflammatory shock in children during COVID-19 pandemic. Lancet. 2020; 395: 1607-1608.

13. Toubiana J, Poirault C, Corsia A et al. Kawasaki-like multisystem inflammatory syndrome in children during the covid-19 pandemic in Paris, France: prospective observational study. BMJ. 2020; 369: m2094.

14. Wang $\mathrm{F}$, Nie J, Wang $\mathrm{H}$ et al. Characteristics of peripheral lymphocyte subset alteration in COVID-19 pneumonia. The Journal of Infectious Diseases. 2020; 221: 1762-1769.

15. Bordoni V, Sacchi A, Cimini E et al. An inflammatory profile correlates with decreased frequency of cytotoxic cells in COVID-19. Clin Infectious Dis. 2020; 71 (16): 2272-2275.

16. Cabrero-Hernandez M, Garcia-Salido A, Leoz-Gordillo I et al. Severe SARS-CoV-2 infection in children with suspected acute abdomen: a case series from a tertiary hospital in spain. Pediatr Infect Dis J. 2020; 39 (8): e195-e198.

17. Henderson LA, Canna SW, Schulert GS et al. On the alert for cytokine storm: Immunopathology in COVID-19. Arthritis Rheumatol. 2020; 72: 1059-1063. 
18. Lou B, Li TD, Zheng SF et al. Serology characteristics of SARS-CoV-2 infection since exposure and post symptom onset. Eur Respir J 2020; 56: 2000763.

19. Tagarro A, Epalza C, Santos $M$ et al. Screening and severity of coronavirus disease 2019 (COVID-19) in children in Madrid, Spain. JAMA Pediatr. 2021; 175 (3): 316-317.

20. Sethuraman N, Jeremiah SS, Ryo A. Interpreting diagnostic tests for SARS-CoV-2. JAMA. 2020; 323 (22): 2249-2251.

21. Feldstein LR, Rose EB, Horwitz SM et al. Multisystem inflammatory syndrome in U.S. children and adolescents. N Engl J Med. 2020; 383: 334-346.

22. Barrios TA, Centeno MF, Rojo SH et al. National consensus on the cardiological treatment and follow-up of Kawasaki disease. Anales de Pediatria. 2018; 89: 188e1-e22.

23. Davies P, Evans C, Kanthimathinathan HK et al. Intensive care admissions of children with paediatric inflammatory multisystem syndrome temporally associated with SARSCoV-2 (PIMS-TS) in the UK: a multicentre observational study. The Lancet Child \& Adolescent Health. 2020; 4 (9): 669-677.

24. Documento de manejo clínico del paciente pediátrico con infección por SARS-CoV-2 en cuidados intensivos. 2020. [Accesado 30 Junio 2020] Disponible en: https://secip. com/wp-content/uploads/2020/05/Protocolo-de-manejocl\%C3\%ADnico-COVID-19-en-UCl-20200518.pdf.

25. Verbeek JH, ljaz S, Mischke $\mathrm{C}$ et al. Personal protective equipment for preventing highly infectious diseases due to exposure to contaminated body fluids in healthcare staff. The Cochrane Database of Systematic Reviews. 2016; 4: CD011621.

26. Cinesi GC, Peñuelas RO, Luján TM et al. Clinical consensus recommendations regarding non-invasive respiratory support in the adult patient with acute respiratory failure secondary to SARS-CoV-2 infection. Arch Bronconeumol. 2020; 56 (Suppl 2): 11-18.

27. Pediatric Acute Lung Injury Consensus Conference G. Pediatric acute respiratory distress syndrome: consensus recommendations from the Pediatric Acute Lung Injury Consensus Conference. Pediatr Crit Care Med. 2015; 16: 428-439.

28. Ramcharan T, Nolan O, Lai CY et al. Paediatric inflammatory multisystem syndrome: temporally associated with SARSCoV-2 (PIMS-TS): cardiac features, management and shortterm outcomes at a UK Tertiary Paediatric Hospital. Pediatr Cardiol. 2020; 41 (7): 1391-1401.

29. Clinical Guidance for Pediatric Patients with Multisystem Inflammatory Syndrome in Children (MIS-C) Associated with SARS-CoV-2 and hyperinflammation in COVID-19.
2020. [Accessed 23 June, 2020] Available in: https://www. rheumatology.org/Portals/0/Files/ACR-COVID-19-ClinicalGuidance-Summary-MIS-C-Hyperinflammation.pdf.

30. Mehta P, Cron RQ, Hartwell J, Manson JJ, Tattersall RS. Silencing the cytokine storm: the use of intravenous anakinra in haemophagocytic lymphohistiocytosis or macrophage activation syndrome. Lancet Rheumatol. 2020; 2 (6): e358-e367.

31. Guaraldi G, Meschiari M, Cozzi-Lepri A et al. Tocilizumab in patients with severe COVID-19: a retrospective cohort study. Lancet Rheumatol. 2020; 2 (8): e474-e484.

32. Nozawa T, Imagawa T, Ito S. Coronary-artery aneurysm in tocilizumab-treated children with Kawasaki's disease. N Engl J Med. 2017; 377: 1894-1896.

33. Bhimraj A, Morgan RL, Shumaker AH et al. Infectious Diseases Society of America Guidelines on the treatment and management of patients with COVID-19. Clin Infect Dis. 2020; ciaa478.

34. Sarma $\mathrm{P}$, Kaur $\mathrm{H}$, Kumar $\mathrm{H}$ et al. Virological and clinical cure in COVID-19 patients treated with hydroxychloroquine: a systematic review and meta-analysis. J Med Virol. 2020; 92: 776-785.

35. Tang W, Cao Z, Han M et al. Hydroxychloroquine in patients with mainly mild to moderate coronavirus disease 2019 : open label, randomised controlled trial. BMJ. 2020; 369: m1849.

36. Cavalcanti AB, Zampieri FG, Rosa RG et al. Hydroxychloroquine with or without azithromycin in mild-tomoderate covid-19. N Engl J Med. 2020; 383: e119.

37. Rosenberg ES, Dufort EM, Udo T et al. Association of treatment with hydroxychloroquine or azithromycin with inhospital mortality in patients with COVID-19 in New York State. JAMA. 2020; 323 (24): 2493-2502.

38. Wang $Y$, Zhang D, Du G et al. Remdesivir in adults with severe COVID-19: a randomised, double-blind, placebocontrolled, multicentre trial. Lancet (London, England). 2020; 395: 1569-1578.

39. Shekar K, Badulak J, Peek G et al. Extracorporeal life support organization coronavirus disease 2019 interim guidelines: a consensus document from an international group of interdisciplinary extracorporeal membrane oxygenation providers. ASAIO J. 2020; 66 (7): 707-721.

Correspondencia:

Alfredo Tagarro

Hospital Universitario Infanta Sofía

28702 San Sebastián de los Reyes, Madrid, España.

E-mail: alfredotagarro@gmail.com 